\title{
MEDICAL IMAGE - BASED COMPUTATIONAL MODEL OF PULSATILE FLOW IN SACCULAR ANEURISMS
}

\author{
Stéphanie Salmon ${ }^{1}$, Marc Thiriet ${ }^{2}$ and Jean-Frédéric Gerbeau ${ }^{3}$
}

\begin{abstract}
Saccular aneurisms, swelling of a blood vessel, are investigated in order (i) to estimate the development risk of the wall lesion, before and after intravascular treatment, assuming that the pressure is the major factor, and (ii) to better plan medical interventions. Numerical simulations, using the finite element method, are performed in three-dimensional aneurisms. Computational meshes are derived from medical imaging data to take into account both between-subject and within-subject anatomical variability of the diseased vessel segment. The $3 \mathrm{D}$ reconstruction is associated with a faceted surface. A geometrical model is then obtained to be finally meshed for a finite element use. The pulsatile flow of incompressible Newtonian blood is illustrated by numerical simulations carried out in two saccular aneurism types, a side- and a terminal-aneurism. High pressure zones are observed in the aneurism cavity, especially in the terminal one.
\end{abstract}

Mathematics Subject Classification. 68U05, 68U10, 76D05, 35Q30, 65N30.

\section{INTRODUCTION}

Large-artery diseases as aneurisms (local dilation of a blood vessel) or stenoses (local narrowing of the vessel lumen due to the development of atheromatous plaques) are a major cause of death in western societies. Since haemodynamical factors play a major role both in the lesion genesis and evolution, numerical tests of local blood flow behaviour during a whole cardiac beat are needed. The present work deals with saccular aneurisms. The aneurism is located either on the vessel edge, and then called side aneurism, or at a branching region, and called lateral or terminal whether the vessel trunk gives birth to a lateral branch or divides into two main daughter vessels. Whereas cerebral aneurism may result from congenital defects, other aneurisms, very often, develop from a lesion in the structural layer of an artery, like atherosclerosis, or are caused by vessel wall infections or traumas. The vessel wall is subjected to a plastic deformation and may become thinner and weaker than the normal artery wall; consequently, wall rupture can occur. Unruptured aneurisms may be discovered when they cause symptoms, which depend on the location and size of the aneurism. Sometimes, they are diagnosed when they rupture. Finally, aneurisms are also found by angiography.

Keywords and phrases. 3D surface reconstruction, finite element method, medical images, mesh generation, Navier-Stokes equations.

${ }^{1}$ UFR de Mathématique et d'Informatique, Université L. Pasteur, 67084 Strasbourg, France.

2 Projet INRIA "BANG" and Laboratoire Jacques-Louis Lions, CNRS UMR 7598, UPMC, 75252 Paris, France.

${ }^{3}$ INRIA, Projet BANG, BP 105, 78153 Le Chesnay, France. e-mail: jean-frederic.gerbeau@inria.fr 
X-ray and MR scanning provide the aneurism's size and location, as well as adjoining organs and vessels. Saccular aneurisms are classified into three categories according to the largest width $w_{\mathrm{a}}$ of the aneurism sac (small, large $-12 \leq w_{a} \leq 25 \mathrm{~mm}$ - and giant). Other geometrical characteristics are given by the neck size, the pouch shape and the angle between the greatest aneurismal length and the main-flow local direction. The geometrical features, especially the sac and orifice sizes, are important short-term prognosis factors, because they affect the treatment quality.

Two main therapies may be applied to saccular aneurisms. The first treatment consists in placing a small metallic clip across the base of the aneurism whereas the normal artery wall is reconstructed to maintain blood flow to the irrigated tissues. The alternative to the surgical operation is the percutaneous, minimally invasive image-guided endovascular procedure. This treatment reduces the surgical risks, is less expensive and is equally or more efficient in treatment than the surgical one. A catheter is threaded from a peripheral artery down to the aneurism site, and, under X-ray guidance, a metallic coil is placed into the aneurism cavity. Once the aneurism cavity is filled with coils, a foreign body, the blood in the aneurism clots. The aneurism cavity is, very often, partially filled because the coils are compressed by the blood flux which enters in the cavity. Moreover, the thrombi on the coils retract. The medical staffs using catheter-based procedures for implantation of medical devices in the diseased vascular region, require thus (1) an appropriate visualisation of the diseased vascular region and its surroundings, (2) a determination of local flow indices and flow disturbances, (3) a planification tool for the treatment strategy, which minimises the complication risk, and (4) a check-up to investigate the medical device effects on local blood flow and to estimate the short-term prognosis and to possibly project long-term evolution of the treated vessels during more or less frequent medical supervision.

Two different saccular aneurisms, a side - and a branching lesions, illustrate the sequence of operations used to compute the blood flow in the diseased vascular region. Different stages lead to the computational mesh. The first stage consists in three-dimensional reconstruction of the in vivo vessel anatomy. The associated faceted surface is then transformed into a geometrical model suitable for computation purpose. Once an appropriate domain mesh is obtained, numerical simulations are performed to describe both the velocity and pressure fields at different phases of the cardiac cycle and provides haemodynamical data to the medical staff.

\section{MEthoD}

\subsection{Mesh generation}

Geometrical input data are provided by 3D surface, point set or vascular contours get by medical image processing. Two main acquisition techniques are the sources of medical images, magnetic resonance (MRA) and X-ray computed tomography (CTA) angiography. In MRA, the released electromagnetic energy after radio wave exposure in a strong magnetic field is measured and analyzed to form two- or three-dimensional images. CTA uses a special X-ray equipment and an intravenous contrast injection to produce cross-sectional blood vessel images.

\subsubsection{Investigated aneurisms}

Cerebral aneurism. The first kind (A1) of saccular aneurism is a congenital cerebral aneurism located at the apex of the bifurcation of a branch of the middle cerebral artery; it is thus called terminal aneurism (Fig. 1). This large aneurism has the following main geometrical features: the cavity is biloculated (with a very long loculus, on the left in Figure 1, called below the nose region). The inlet section of the neck, defined as the channel between the vessel and the actual cavity, takes the place of both the bifurcation apex and the entrance short segment of the outer edges (with respect to the local center of the vessel curvature) of both terminal artery branches. The aneurism cavity (or pouch or sac) is facing the curved stem; the blood impacts the aneurism wall. The neck is badly defined and may be assumed to be axially short and transversally rather broad.

The 3D facetisation is associated with a 3D isodensity surface, which is processed from high quality medical images. The marching-cube approach, based on a "voxelhood" analysis, extracts, after a necessary step of thresholding, a 3D isodensity surface from the image set [15]. Artefacts are generated due to the lack 

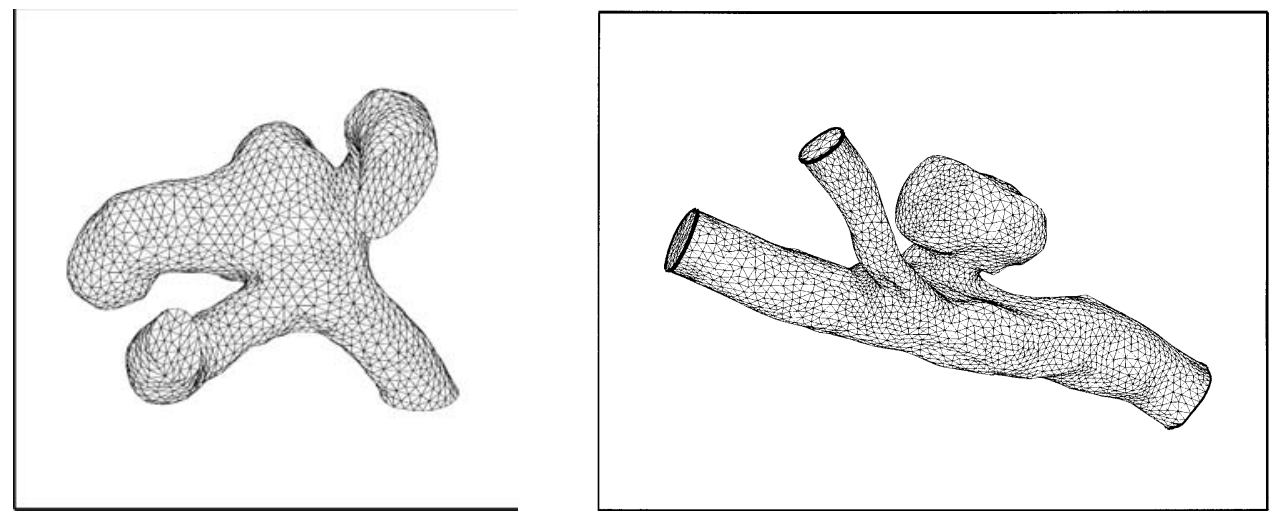

Figure 1. Left, the saccular terminal aneurism. Right, the saccular side aneurism.

in significant threshold. Besides, the signal provided by the flowing blood in the vessel lumen is heterogeneous. A smoothing stage based on a bi-Laplacian operator is required to remove the "staircase" artefacts [21]. The region of interest is too large; a smaller exploration volume is thus chosen using arbitrary planes. The resulting exploration volume is composed of a $3 \mathrm{D}$ curved trunk, its diseased bifurcation and the entrance region of both curved branches.

Iliac aneurism. The second kind (A2) of saccular aneurism is a probably accidental aneurism located at the outer edge of the common iliac artery, slightly upstream from a branching segment (Fig. 1). The small aneurism has a well-defined neck which appears as a narrow and short channel conveying the blood into the aneurismal cavity (the neck is narrow in the direction normal to the local vessel axis, and larger, although not very wide, in the direction parallel of the local vessel axis). Although of small size, the iliac aneurism induces a compression of the branch, which can be better seen after 3D reconstruction. Three models have been derived from image processing, using a contour detection algorithm (Fig. 2). The vascular network of interest is composed of the downstream segment of the aorta, of its bifurcation into two common iliac arteries and a rather long upstream segment of these two branches. The diseased common iliac artery is longer than the normal one and presenting a branching segment (Fig. 3).

Model I (A2M1) is composed of the diseased artery with its side aneurism, the branching region and the entrance segments of both branches (on the right in Fig. 1). The reconstruction of the 3D surface has been made after a level-set based image processing, using a spline-fitting method associated with a CAD software [16].

Model II (A2M2) and III (A2M3) correspond to the whole explored network. Both are obtained using home-made softwares dedicated to surface reconstruction from planar parallel contours (Fig. 3). For A2M2, the space discretization starts by a slice-couple treatment [3]. To fit up a surface on a set of contours amounts to construct a volume enclosed by these contours. The global volume is considered as the union of independent pieces resulting from pair treatment. The in-house package "Nuage" produces such a volume, dealing with one pair of consecutive contours at a time [2]. A triangulated surface is drawn in between each pair of consecutive sections, ensuring that each point in a contour is connected to its closest point in the next contour. Once a 2DDelaunay triangulation is determined in each section limited by the image-postprocessed vessel contours of two consecutive slices, tetrahedra are generated in-between section couples by overlaying Voronoï diagrams of the Delaunay triangulations. The reconstructed surface is then the interface between inner and outer tetrahedra. Interesting properties of the method are (i) to ensure a convex reconstruction volume between two convex slices and (ii) to easily handle branch sites. The drawback of this method is to induce more or less great twist of the wall branches.

The points which define the vessel contours are used as input data to mesh A2M3. A node set is derived for each contour after a cubic spline fit and smoothing; the nodes are equally spaced along the vessel contour. Then, two successive slices are projected in a same plane and a 2D constrained Delaunay's triangulation is built. 


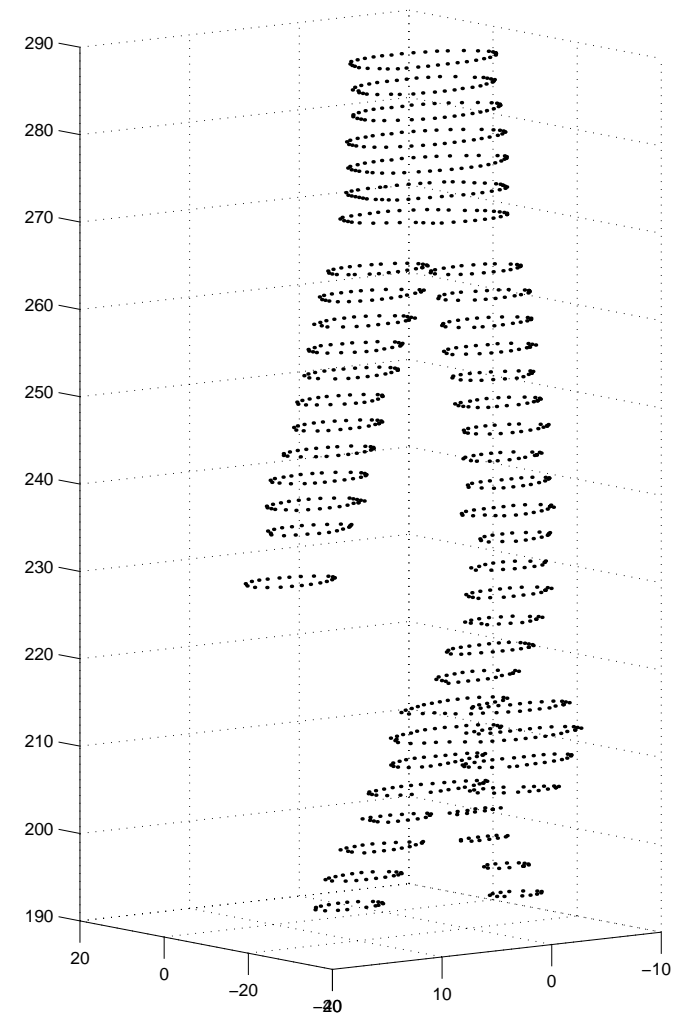

Figure 2. Vessel contours from the set of saved slices from medical imaging.

The surface triangles are then extracted by elevation of the two planes. The entry and exit sections of A2M3 are rotated in order to be normal to the local vessel axis. The disadvantage of this technique is to introduce high curvature branching apex, which less mimic the anatomical branching than A2M2. Both meshing techniques, with their respective advantages and drawbacks, have been used to investigate mesh effect and local features of the vessel geometry on the flow field.

\subsubsection{From faceting to computational meshes}

The triangulations obtained from the images need further treatment before computations. The operation, which leads to a computational mesh from a faceted surface, is composed of three main steps.

Step 1 builds a computation-oriented surface triangulation. It deals mainly with ends of the fluid domain to ensure that end sections are cross sections, where the pressure is assumed to be uniform, without neither transverse pressure gradient secondary to curvature effects nor axial pressure gradient due to vessel section which are non-perpendicular to the vessel axis. The edges of the inlet and outlet sections are labeled. A mesh scan of the facets gives the triangles with a labeled edge, which belong to the vessel wall and not to the vessel section. Once these facets are known, their normals are computed. The local axis, orthogonal to all the normals, is then determined by a least-square method. Sections are thus corrected to be orthogonal to this axis. In order to avoid flow disturbance induced by boundary conditions (BC) close to the exploration volume and to take into account the upstream and downstream effects on the flow of the complex vessel geometry (combination of curved vessels, possible branching segment, wall lesions and slight taper), straight segments in the direction of the local axis are added. The new inlet and outlet cross sections are labeled for $\mathrm{BC}$ assignation.

Step 2 is aimed at constructing surface meshes that match strong requirements related to the accuracy of the surface approximation (geometry) and to the element shape and size quality (computation). Surface remeshing 

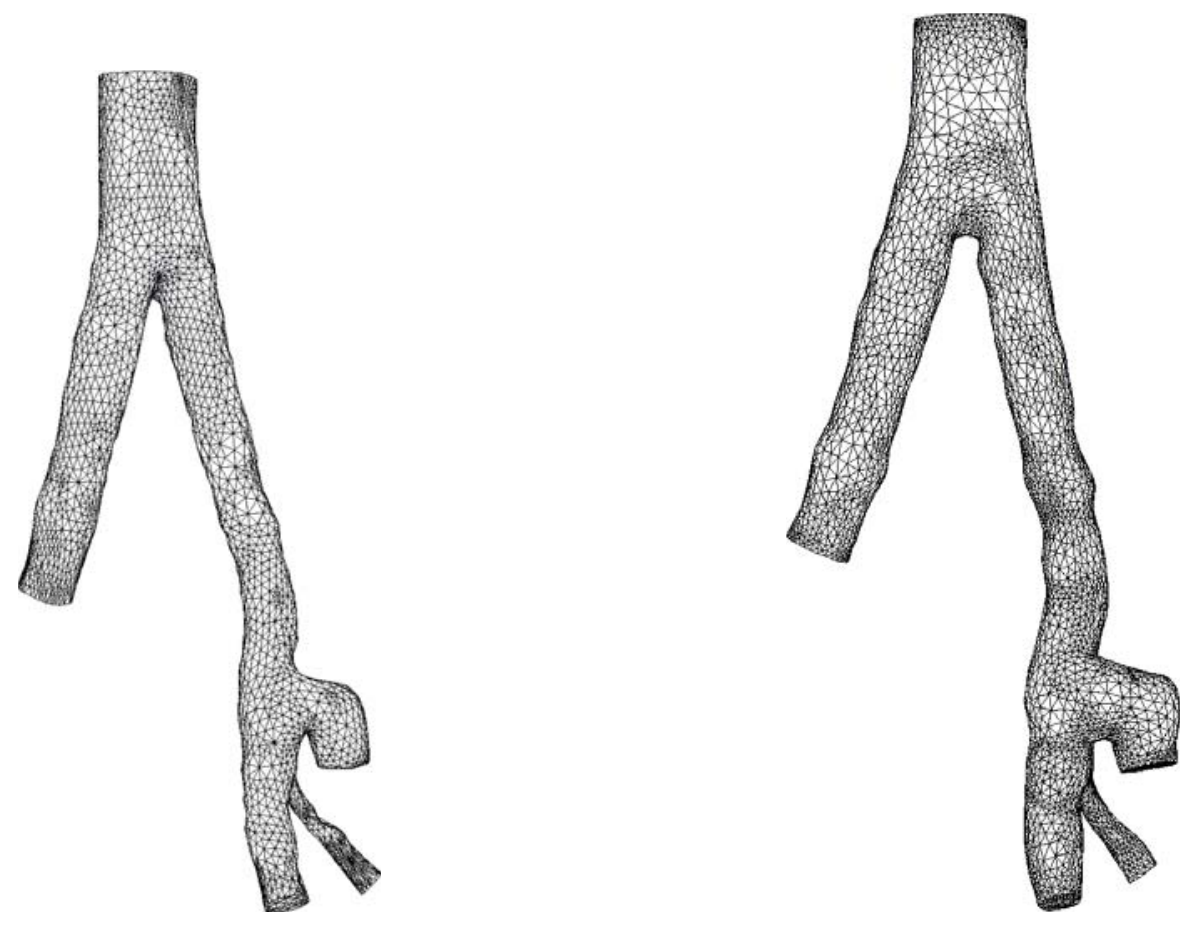

Figure 3. Surface meshes of the whole explored iliac network, A2M2 (left) and A2M3 (right).

TABLE 1. Aneurism-model mesh features.

\begin{tabular}{|c|c|c|c|c|}
\hline & A1 & A2M1 & A2M2 & A2M3 \\
\hline node number & 7910 & 12800 & 10820 & 12550 \\
\hline element number & 36970 & 63360 & 50270 & 58250 \\
\hline
\end{tabular}

uses the INRIA's software YAMS [9]. A compromise is found between the minimal number of triangles of the surface discretization and the preservation of the geometric accuracy of the geometric approximation of the underlying surface. Node reduction must, indeed, be done in order to avoid redundant data, using element quality criteria. Moreover, the geometrical model must be coarsened because the reconstruction algorithm converts the initial sampled data into surface facetisation having the same degree of complexity as the original data. Consequently, the node number is too great. The simplification procedure is based on the Hausdorff distance. This algorithm involves, in particular, vertex deletion and edge flipping. Besides, a smoothing stage is required to remove the step-shaped contours, which are artifacts induced by the image processing. Furthermore, element shapes and sizes must be suitable for numerical tests. Therefore, an anisotropic geometric metric map based on the local principal directions and radii of curvatures is contructed in the tangent planes related to the mesh vertices. This metric map prescribes element sizes proportional to the local curvature of the surface. Mesh enrichment can be finally performed to ensure sufficient quality meshes, especially in region of expected changes in flow properties. In any case, the mesh element density is sufficiently small to limit the computational time for medical use.

Step 3 provides a finite-element triangulation (e.g. [7]). From the surface triangulation the tetrahedron mesh is provided by the fast INRIA's software GHS3D [11]. Node and element numbers are given in Table 1. 


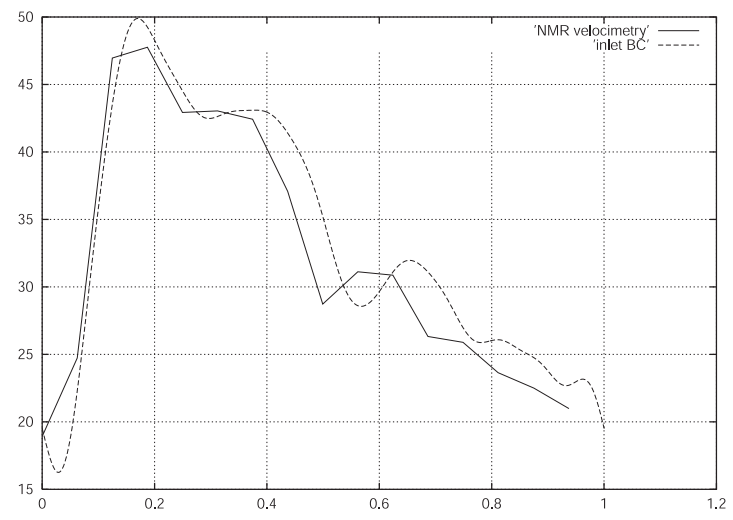

FIGURE 4. Inlet velocity condition: measured signal (continuous line) and Fourier-transform flow rate (dashed line) for prescribed boundary condition.

\subsection{Numerical simulations}

The vessel wall is assumed to be rigid, because the explored deep arteries, much less compliant than other blood vessels, surrounded by an incompressible environment, is confined into the skull. The blood, concentrated suspension of cells, is supposed to be incompressible, homogeneous and Newtonian, because red blood cells (RBC) are assumed neither to have time enough to aggregate (the time constant of RBC agregation is supposed to be greater than the convective time scale) nor to deform in the large blood vessels.

Flow modeling. The used numerical technique to solve the fluid mechanics equations is the finite element method. The basis for the finite element formulation of the set of governing equations is the variational form of the Navier-Stokes equations [13]. The pressure $p$ is defined at the four vertices of the tetrahedron $\left(P_{1}\right.$ element) and the velocity $\mathbf{u}$ at both the vertices and the barycenter $\left(P_{1}\right.$ bubble element $\left.[1]\right)$. The convective term is approximated by the method of characteristics [18]. The solution is obtained via a generalized Uzawapreconditioned-conjugate gradient method [12]. The initial condition is given by a stationary Stokes problem with the same boundary condition as the unsteady one (period $T=1 \mathrm{~s}$ ).

Boundary conditions. The boundary of the fluid domain is partioned into three surfaces: entry cross section $\Gamma_{1}$, exit cross sections $\Gamma_{2}$ and vessel wall $\Gamma_{3}$. The classical no-slip condition is applied to the vessel wall. A time-dependent uniform injection velocity $\mathbf{u}_{\Gamma}(t)$ is prescribed at the inlet, which is obtained from the Fourier transform of the in vivo MR velocimetry signal (Fig. 4). At the outlet cross-sections (perpendicular to the local vessel axis and exits of short straight pipes), constraint is equal to zero. In summary, BCs are: $\mathbf{u}(t)=$ $\mathbf{u}_{\Gamma}(t)$ on $\Gamma_{1}, \quad \boldsymbol{\sigma} \hat{\mathbf{n}}_{2}=\mathbf{0}$ on $\Gamma_{2}, \mathbf{u}=\mathbf{0}$ on $\Gamma_{3}$, where $\boldsymbol{\sigma}$ is the Cauchy stress tensor and $\hat{\mathbf{n}}_{2}$ the outer unit normal vector at $\Gamma_{2}$.

\section{Governing parameters}

A1 model. The stem peak Reynolds number $\widehat{R e}$, based on the peak cross-sectional average velocity $\hat{U}$ and on the trunk radius $R$ at the entrance cross section is equal to about 160 (blood kinematic viscosity $\left.\nu=4 \times 10^{-6} \mathrm{~m}^{2} \mathrm{~s}^{-1}\right)$. The Stokes number, based on the trunk radius at the entrance cross section, $S t=R(\omega / \nu)^{1 / 2} \sim 1.6$. The Strouhal number $\operatorname{Str}=\omega R / \hat{U} \sim 0.02$.

A2 models. The aortic peak Reynolds number $\widehat{R e}$, based on the peak cross-sectional average velocity $\hat{U}$ and on the aorta radius $R$ (entrance cross section of the entire investigated domain A2M2 or A2M3) is equal to about 1110, whereas the iliac peak Reynolds number is equal to about 650 (flow upstream from the aneurism). The Stokes number, based on the aorta radius, $S t \sim 11.2$ and the Strouhal number $S t r \sim 0.11$. 

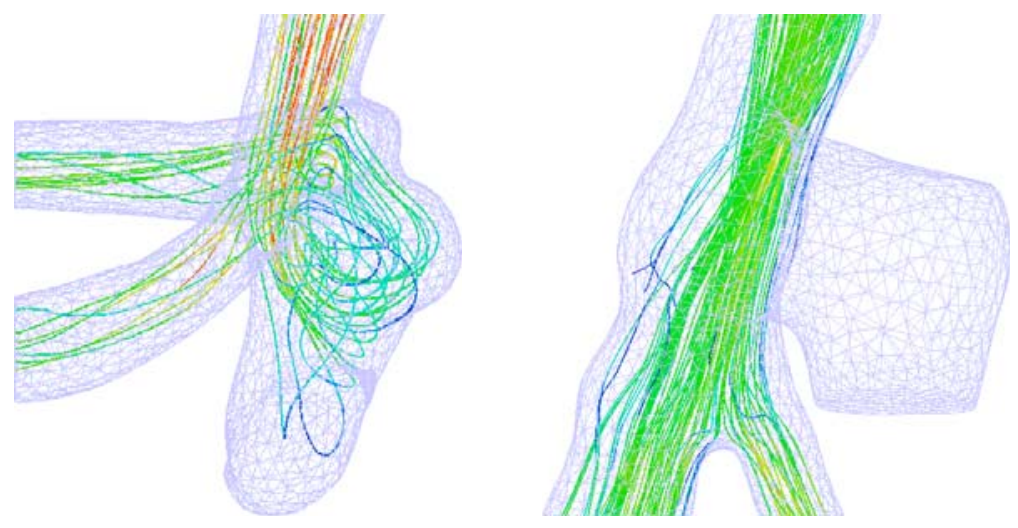

Figure 5. Streamlines in the terminal aneurism (A1 model, left) and in side aneurism (A2M3 model, right) at phase 2 .

\section{Results}

Haemodynamic quantities have been plotted at four selected phases of the cardiac cycle: (i) phase 1 at about $2 / 3$ of the time duration of the accelerating phase, (ii) phase 2 slightly after peak flow, (iii) phase 3 at the beginning of the decelerating phase when the instantaneous flow rate is nearly the same as in phase 1 , and (iv) phase 4 at about $3 / 4$ of the time duration of the decelerating phase. Three-dimensional streamlines have been traced in the whole computational domain (Fig. 5). The pressure and velocity fields have been displayed in selected planes through the trunk, the aneurismal neck and cavity at different selected phases of the cardiac cycle using isocontour plots, vector fields and streamlines. Three kinds of planes have been used and defined as transverse, coronal and sagittal with respect to the aneurism, when its widest face is viewed. Both A1 and A2 present a nose, i.e. a projecting end part of the aneurism sac. The transverse plane crosses either the aneurism neck or the cavity form its nose to its nucha. Transverse planes are normal to the neck axis. The sagittal plane provides slices either through the nose region of the cavity or through both the cavity and the neck, as well as the artery; the aneurism wall in the sagittal plane is then partly bounded by the aneurism dome or fundus. In the coronal plane, the aneurism section is limited by the wall of the nose, of the fundus and of the nucha. Because A1 is characterized by a wide neck, neck section is always displayed in coronal planes, while it can be away from coronal planes crossing both A2 and the diseased artery.

3D streamlines. Streamlines from points located in the entrance cross section are plotted in the 3D domains of A1 and A2M3 at phase 2 (slightly after peak flow) in Figure 5. Due to lesion implantation on the local vessel network, the blood runs widely through the terminal aneurism, whatever the cycle phase, whereas the streamlines issued from the entrance cross section do not travel through the side aneurism. The streamlines avoid the side aneurism whatever the cycle phase although the neck is located at the wall egde near which the maximum velocities are observed, again whatever the cycle phase.

Pressure field. The pressure is normalised $\left(\tilde{p}=p / p_{\max }\right)$; the dimensionless magnitude, which is known up to a constant is not valuable, except for between-model comparisons. Moreover, the pressure notation is simplified in the following sections $(\tilde{p}=p)$. The pressure fields on the $3 \mathrm{D}$ domains are displayed in A1 and A2, with a zoom on the side aneurism, at phase 2, when the pressure is almost maximum in Figures 6 and 7 respectively. In the terminal aneurism, the pressure reaches high values $(p>0.66)$ at different parts: the egde between the left neck wall and the left branch which faces the flow coming from the curved stem, the nose, the dome part in front of the neck and the front face (Fig. 6). In the side aneurism, the pressure is lower than in A1 $(p<0.63$, whatever the aneurism part). Its value does not depend significantly on the size of the computational domain (A2M1 vs. A2M2 or A2M3). Relative pressure difference in coronal planes between A2M2 and either A2M1 or A2M3, the plane pressure field being close in A2M1 to that in A2M3, are equal to $16 \%$. Such a difference, although 

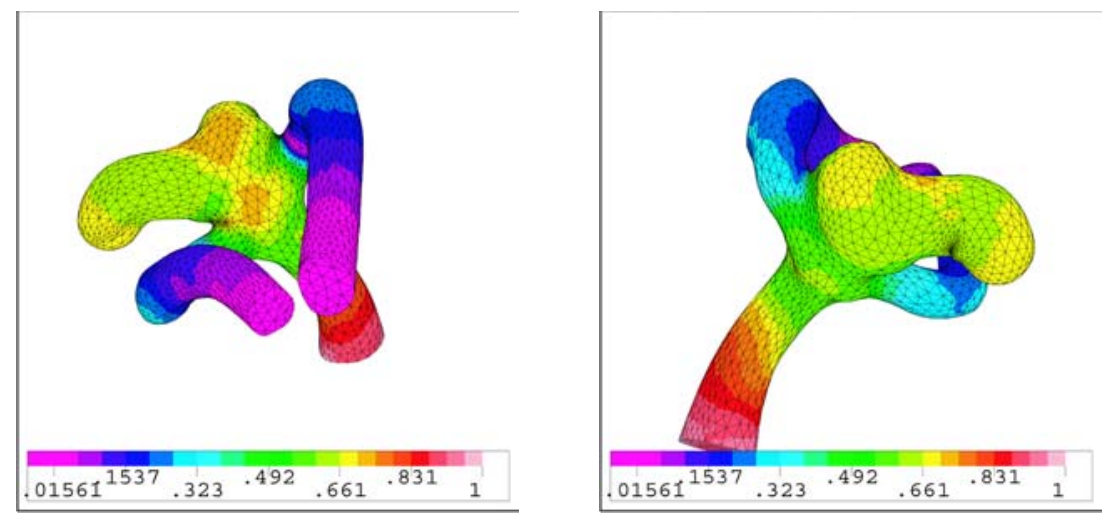

Figure 6. Pressure field in the A1 model at phase 2. Front (left) and rear view (right).
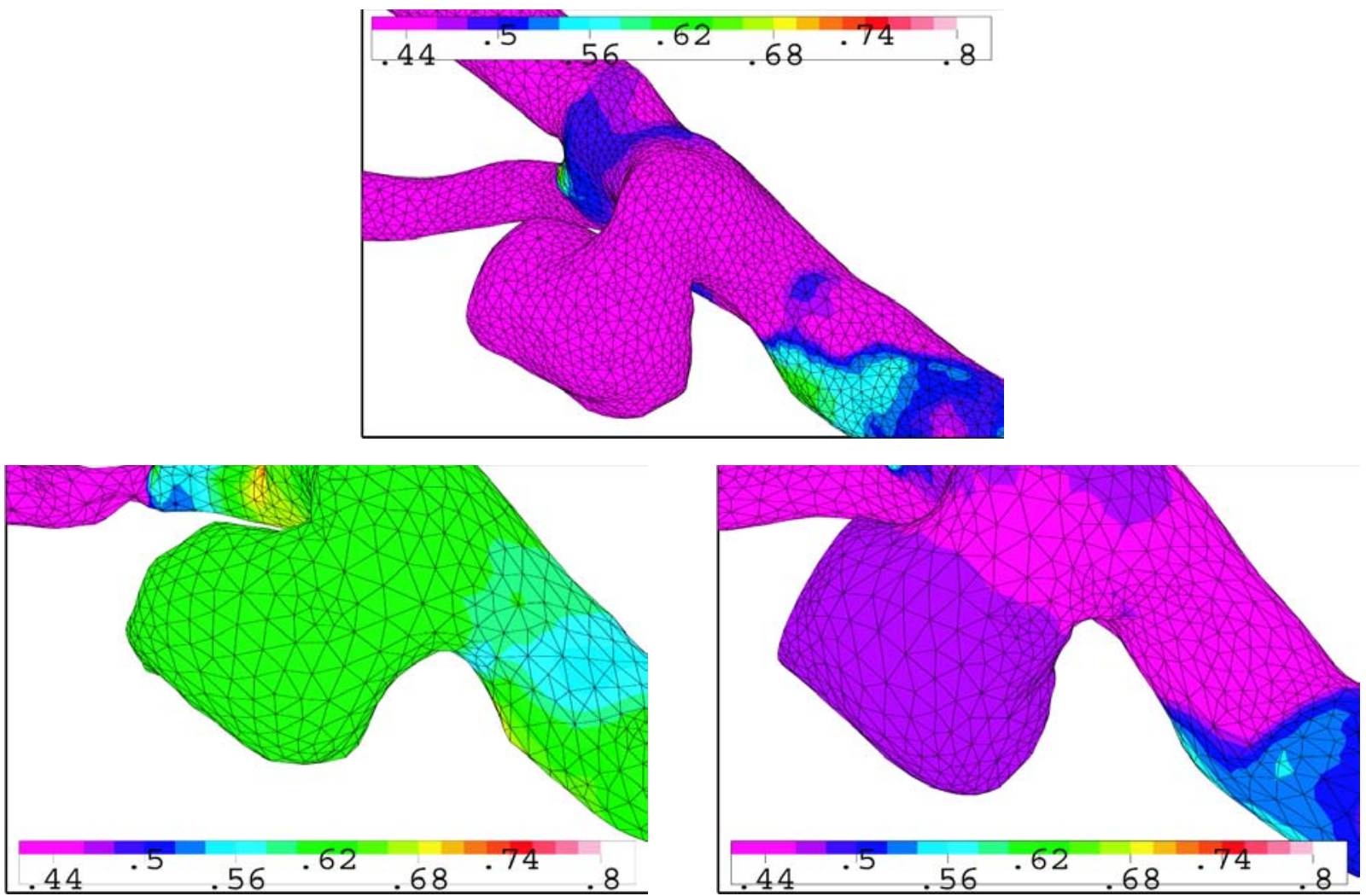

Figure 7. Pressure field in the A2 model at phase 2. Zoom on the aneurism. A2M1 (top), A2M2 (bottom, left) and A2M3 (bottom, right).

significant, can be considered reasonable in the context of multimodelling involved from image acquisition to flow computation. The aneurism pressure is lower in A2M1 $(p<0.45)$. A too short explored segment of the diseased vessel network seems to underestimate the pressure, slightly with respect to the pressure range obtained in A2M3 (Fig. 6). The side aneurisms of the longer computational domains (A2M2 or A2M3) exhibit different local pressure distributions. The pressure is smaller in A2M3 $(p<0.5)$ than in A2M2 $(p<0.63$, Fig. 6$)$. 

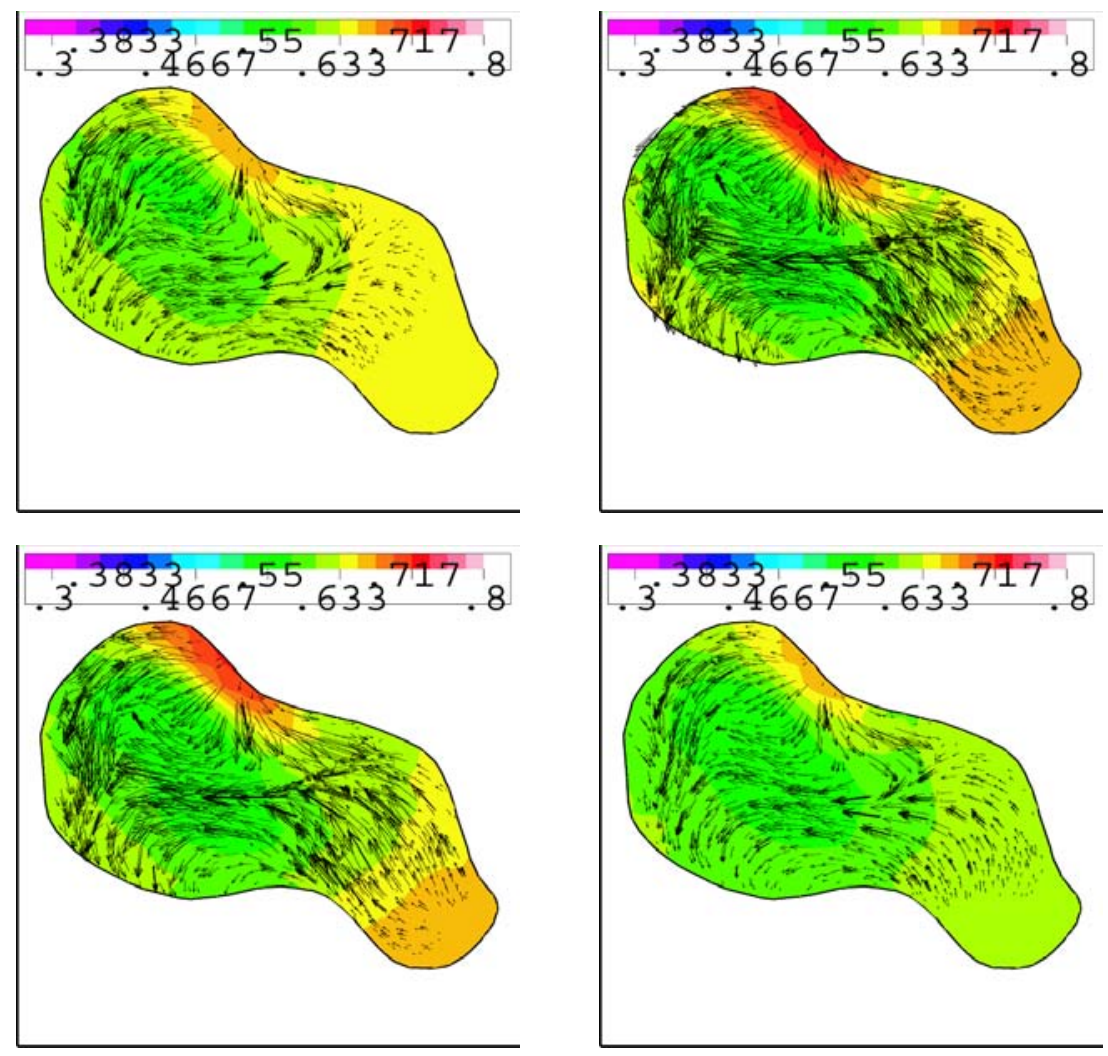

FiguRE 8. Isopressure contours and velocity vectors in A1 transverse plane through mid-height of the sac at the four selected phases from top to bottom and left to right.

The pressure range and the size of high pressure zones vary during the flow cycle (Fig. 8). While the front face of the A1 dome is exposed to high pressure throughout the cycle, $(p \sim 0.65$ at phases 1 and $4, p \sim 0.7$ at phase 2 and 3), the nose is not subjected to high pressure at the end of the deceleration phase.

Figure 9 shows that, in the transverse plane through mid-height of the sac of the side aneurism, the aneurismal pressure is always smaller than 0.5 and the pressure range in this plane is small for the four selected phases ([0.346,0.347] for phase $1,[0.460,0.461]$ for phase $2,[0.447,0.449]$ for phases 3 and 4$)$. The pressure maximum is observed at the aneurism lateral wall ("nucha") in continuity with the upstream aneurism lip (or neck lateral wall opposite to the aneurism "nose").

Velocity field. In the terminal aneurism A1, the streams coming from the core of the transition zone are rotatory within the aneurismal sac to exit along the side walls and the lips into the corresponding branches (Fig. 10). The velocity along the fundus wall is significant especially at phase 2; the fluid does not stagnate. A swirl set occurs in the cavity (Figs. 11 and 12).

The isovelocity contours in the sagittal plane through the neck and sac of the side aneurism confirm that the high velocity region in the diseased vessel is located near the bulged wall (Fig. 13). Contrary to the terminal aneurism, fluid currents are not strong as revealed by the velocity magnitude using isovelocity contours and velocity vectors. 

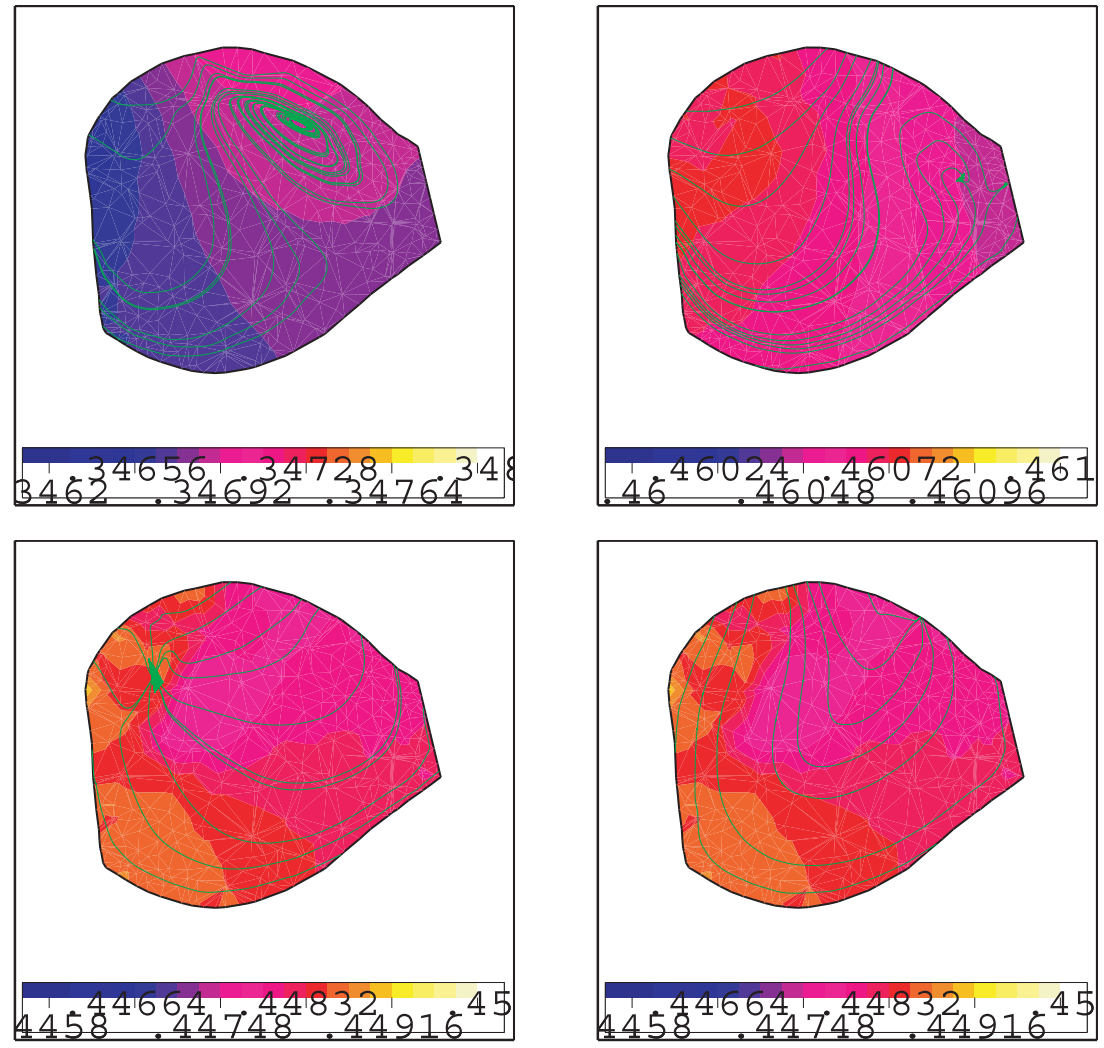

FiguRE 9. Isopressure contours and streamlines in A2M3 transverse plane through mid-height of the sac at the four selected phases from top to bottom and left to right.
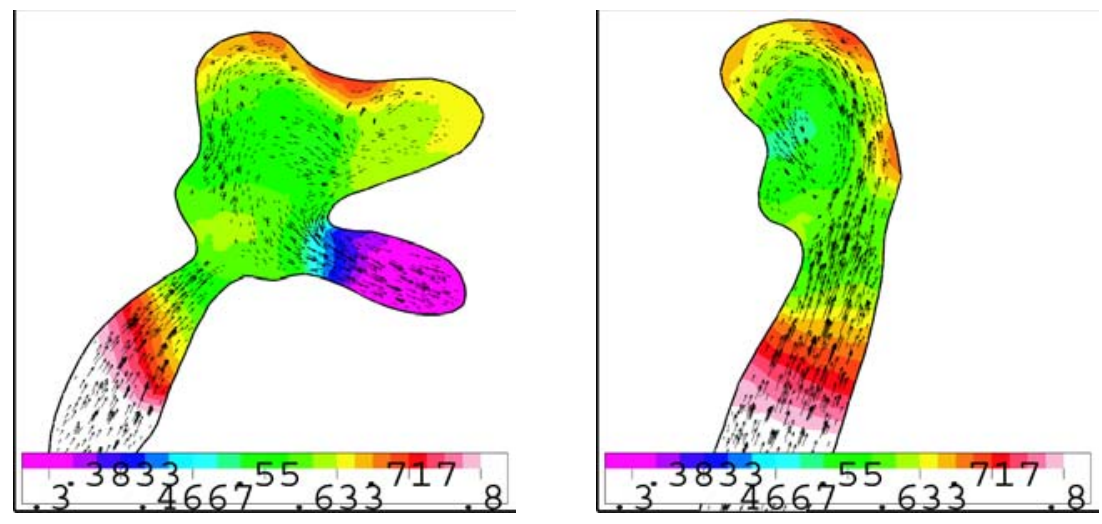

FiguRE 10. Isopressure contours and velocity vectors in A1 coronal (left) and sagittal plane (right) at phase 2 . 

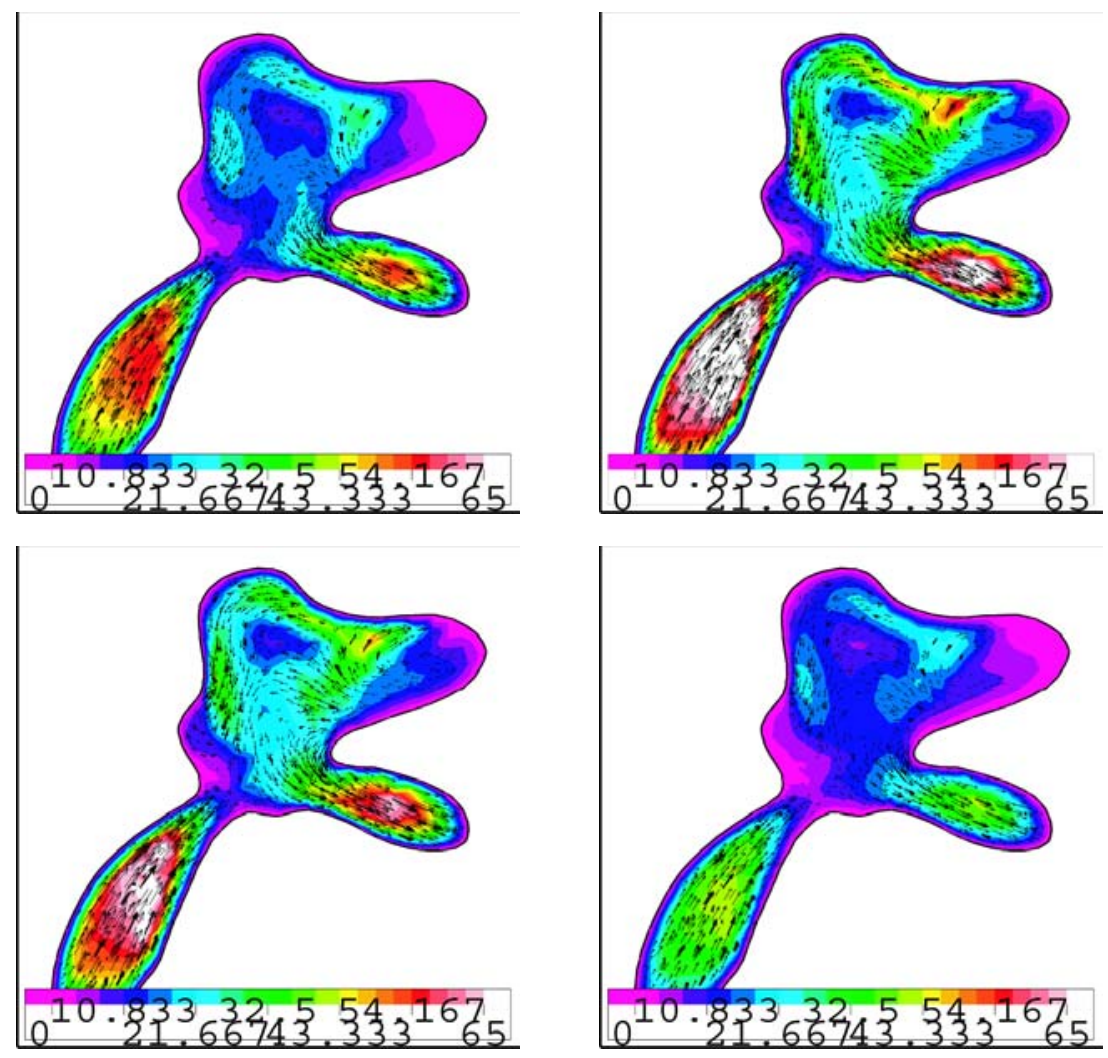

FIGURE 11. Isovelocity contours and velocity vectors in A1 coronal plane at the four selected phases from top to bottom and left to right.

\section{Discussion}

Fluid properties. The blood is a suspension of cells and macromolecules. In the large arteries, the ratio between the vessel length scale and the particle characteristic size is such that the blood is considered as a continuous homogeneous medium. The red blood cells (RBC) play a major role on the rheological behaviour of the blood due to both their size and number. The blood has, in steady state conditions, a shear-thinning behaviour [5]. The sigmoid relationship between the shear rate $\dot{\gamma}$ and the fluid viscosity $\mu$ is explained by RBC agregation at low shear rates associated to high viscosity values in the one hand while RBC deformation and orientation lead to a low viscosity plateau $\mu_{\infty}$ at high shear rates $\dot{\gamma} \geq \sim 10^{2} \mathrm{~s}^{-1}$ (Newtonian behaviour). There is a huge between-subject variability of the $\mu$ vs. $\dot{\gamma}$ relationship. The viscosity maximum $\mu_{0}$, which is difficult to measure with available experimental devices, is about 10 times greater than $\mu_{\infty}$ in steady states. When both $\mathrm{RBC}$ agregation and deformation are inhibited, $\mu_{0}$ is close to $\mu_{\infty}[6]$.

In large arteries, the red blood cells, expelled from the heart cavities where they have been shaked, are expected neither to deform nor to agregate owing to a short convection time scale with respect to the characteristic agregation time. However, in the low flow region of the aneurism cavity along the dome wall where the residence time of particles and conveying cells is much greater, the red blood cell agregation time can be supposed to have the same order of magnitude than the local flow time scale. Non-Newtonian fluid flow must then be simulated. However, as far as we know, dynamical data which suit blood in motion are not yet available. Furthermore, the generalized Newtonian model used to mimic the supposed shear-thinning blood behaviour does not take 

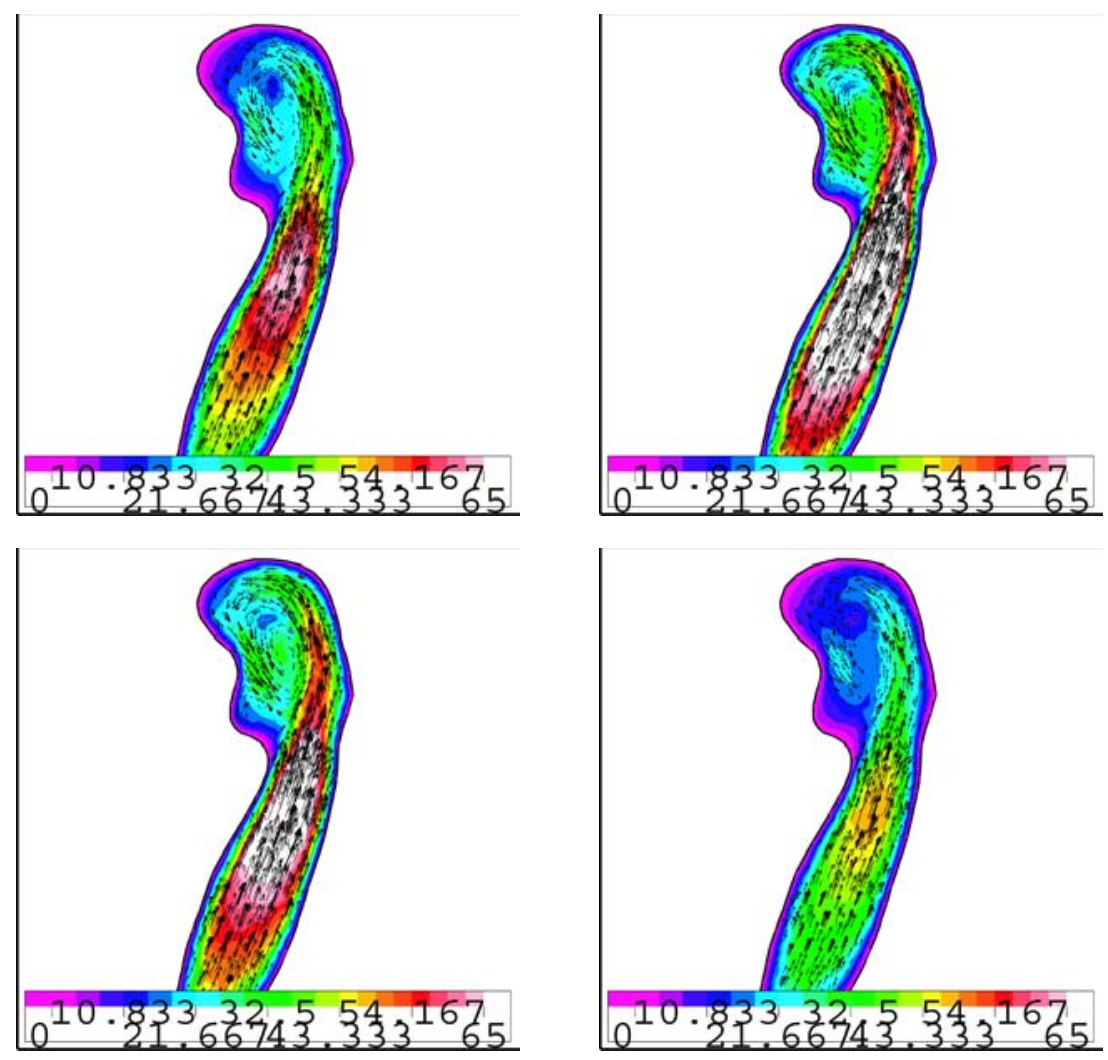

FIGURE 12. Isovelocity contours and velocity vectors in A1 sagittal plane at the four selected phases from top to bottom and left to right.

into account the rheological history of the fluid. Furthermore, the input rheological data are provided by experimental results which are obtained in steady state conditions, far from the physiological ones in the arteries (e.g. [22]). Consequently, the blood is considered as incompressible and Newtonian.

Wall properties. The wall lesion is assumed to be in a steady state, after a long history of plastic deformations. The aneurism wall is much less distensible than the cerebral artery wall [8] and the vessel wall, embedded in its surrounding incompressible tissues, to be much more rigid than the wall of isolated arteries. Moreover, the fluid domain geometry has been found to be the main factor which affects the aneurismal flow much more than the wall deformability [19]. The wall is then assumed to be rigid. However, coupling with wall mechanics will be necessary in the future to check the location of within-wall high stress concentration susceptible to induce wall rupture.

Boundary conditions. A velocity condition is chosen rather than a pressure condition at the inlet section because the velocity can be non-invasively measured. Blood velocity can be measured in vivo by Doppler ultrasound velocimetry in superficial vessels only; MR velocimetry is used for both in superficial and deep vessels. When the lumen size is large enough, the distribution of the three velocity components in the Cartesian frame associated with the scanner can be assessed (e.g. [23]). However, the mid-size arteries, which are often sites of aneurisms, are too small with respect to scanner spatial resolution. The measurements are performed in a sufficiently large artery, often far downstream from the wall lesion. Besides, because of the very tortuous path of the arteries, the cross section cannot be accurately determined. The slice orientation is indeed defined on a $2 \mathrm{D}$ projection image and remains unknown with respect to the local vessel axis. Consequently, either the velocity 

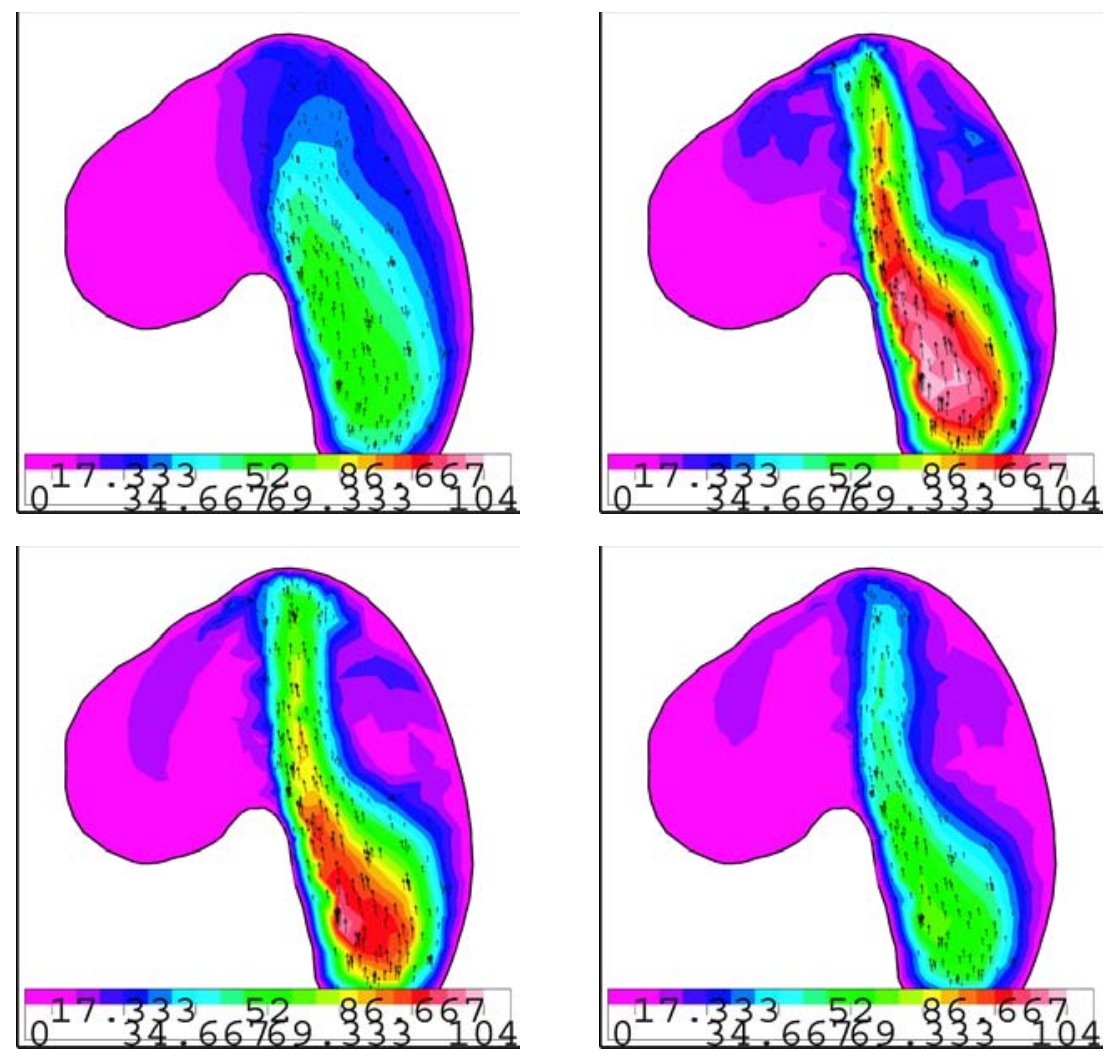

FIGURE 13. Isovelocity contours and velocity vectors in A2M3 sagittal plane at the four selected phases from top to bottom and left to right.

cannot be measured due to resolution limitation or it can only be estimated in larger vessels because (i) cross currents, which are not negligible in the highly curved vessels, cannot be assessed and (ii) the direction of the measured component does not remain constant through the slice thickness. Slice average velocity is measured rather than the velocity distribution in artery sections in order to avoid too great quantification errors.

The so-called Womersley solution, which is the analytical solution for time-dependent flow induced by a pulsatile (non-zero mean) or oscillating (zero mean) input pressure (with one - sinusoidal flow - or several harmonics) corresponding to Poiseuille flow for steady motions, implies a fully developed laminar flow without body forces in a long smooth straight pipe of circular cross-section, a set of properties never encountered in blood circulation and vessel geometry. Such inlet BC is thus not more appropriate than the time-dependent uniform injection velocity used in the present study. However, the uniform velocity condition is associated with high wall shear at the entrance susceptible to induce larger flow separation if adverse pressure gradient is set up, in particular in the transition zone between trunk and branches (The cross mesh is sufficiently coarse to avoid velocity discontinuity between zero Dirichlet wall and inlet BCs). Besides, high entry wall vorticity diffusion and convection in the vessel may rise the swirling amount found in the aneurism cavity.

The usual stress-free outlet BC, associated with assumed equal pressure at branch exits shows the lack of input data. The region of interest, with its aneurism, is characterised by input and output impedances determined by the vascular network upstream and downstream from the detailled 3D explored domain. Multiscale modeling might thus be added to ensure appropriate BCs.

Time periodicity. Successive velocity profiles at a given cycle phase near peak flow and from an arbitrary nucha-to-nose line of a transverse plane through A1 at about mid-height of the aneurism sac have been plotted 


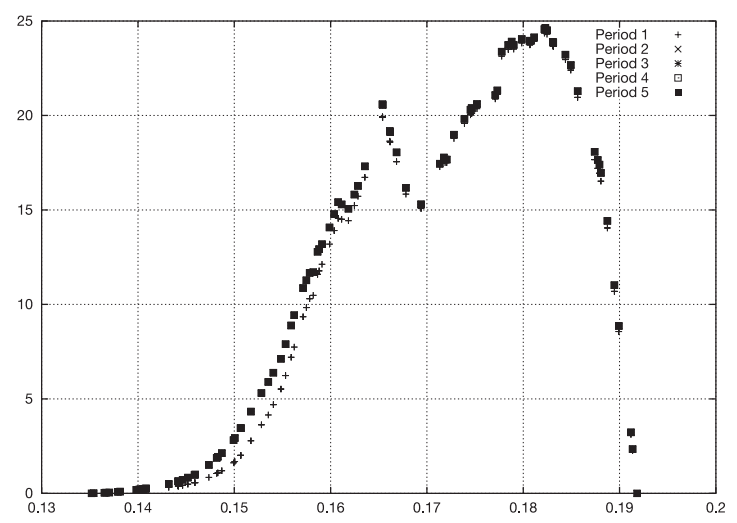

FIGURE 14. Velocity profiles at nearly the peak flow at five successive periods on an arbitrary line of a transverse plane through the aneurismal cavity of the cerebral aneurism.

in Figure 14. Such reproducibility has been observed for flow numerical simulations carried out in different domains based on medical imaging. In two different plane stations of a vessel, the flow, the $L^{2}$ norm of the sectional integrated value of both the velocity and the velocity curl are shown to be wholly superimposed from the first period in an oscillatory flow, or, more precisely, a zero-mean sinusoidal flow. A greater harmonic content of the flow signals might explain that the superimposition of flow variables is only obtained from the second period. Due to slight differences in the velocity magnitude at given spaces and cycle phases between the two first periods, the first period can be considered to give a representative flow. Such a result can be explained by computation initialization using a Stokes problem rather than other initial conditions, as potential flows. The effect of the initialization Stokes mode is damped from the first period.

Meshes. Because such investigations are aimed at guiding busy physicians for diagnosis, treatment planification and prognosis, the mesh element number is choosen to be sufficiently low keeping the domain geometrically representative, in order to provide numerical results as quickly as possible. Besides, bulk flow properties have been shown to remain unchanged in straight collapsed tubes of uniform properties, when the tetrahedron number is multiplied by a factor of 4 [25].

The shape and size of both the aneurism sac and neck is highly dependent on the 3D reconstruction procedure. A2M1 provides a better nucha edge while A2M3 gives a better nose front edge. The ratio between the neck width and the sac width is better in A2M2, whereas A2M1 gives a better aneurism height-to-width ratio. Furthermore, the size of the explored vascular region is expected to affect the pressure within the aneurism.

Although the vessel network modeled in A2M1 is much smaller than in A2M3, the pressure fields are similar. The relative difference in normalized pressure maximum between A2M1 and A2M3, characterised by a smoother vessel configuration than A2M2, is nearly equal to 5\%. The additional upstream bifurcation do not induce significant differences in computational results. On the contrary, the configuration of the computational domain, which model the same region of the vessel network, affects the field of the haemodynamic quantities.

The explored vessel network is the same in A2M2 and A2M3. Isopressure contours plotted on the entire domain of both A2M2 and A2M3 show that the pressure fields are qualitatively similar. However, the local pressure depends on the fluid domain downstream from the trunk (the aorta) bifurcation (Fig. 15). When walldisplayed pressure fields are compared, it appears that the vessel geometry affects the pressure loss, in particular in the aneurism region. Significant pressure differences in the aneurism (16\%) are observed between A2M2 and A2M3. However, these pressure differences can be considered reasonable in the context of multimodelling from image acquisition to 3D reconstruction, then, to meshing and, finally, to flow computation. Moreover, $3 \mathrm{D}$ reconstruction is made from partially saved data and, thus, between-technique differences are maximized. Nevertheless, the 3D reconstruction and meshing must be carefully handled for medical practice. 


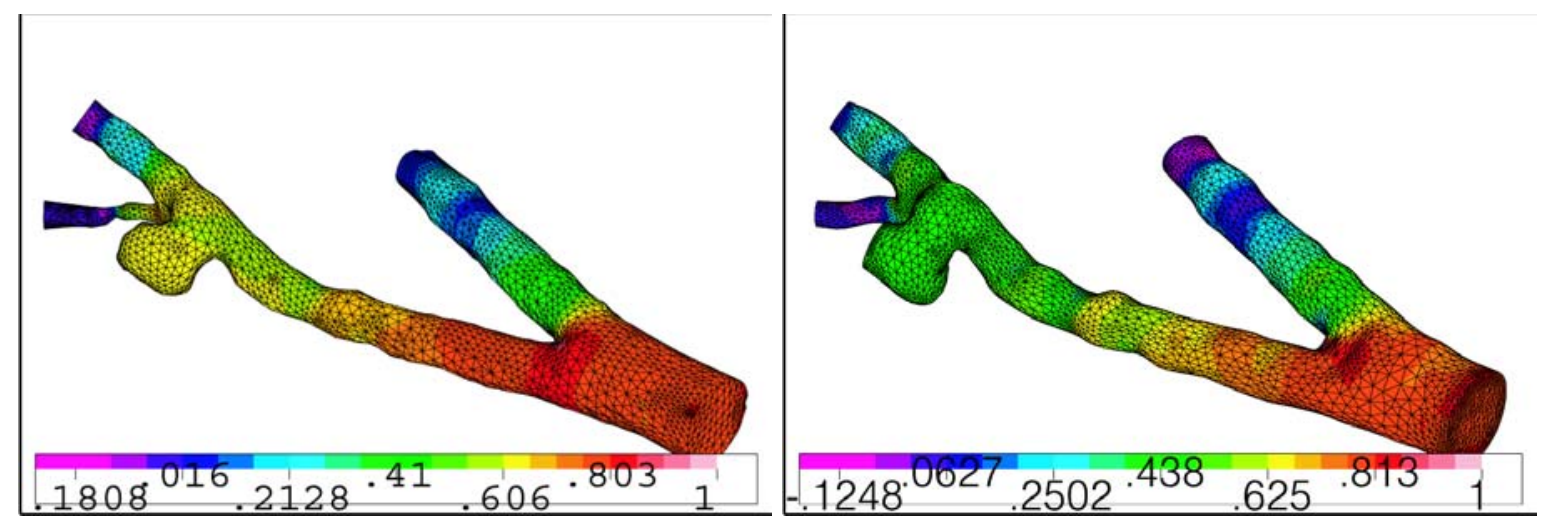

Figure 15. Pressure field in the A2 model at phase 2. A2M2 (left) and A2M3 (right).

The stage of geometrical modeling is then crucial. The faceting associated with isosurface processing of the medical images (model A1) gives faster geometrical models with artefacts; it needs a complete data set and a high-quality image acquisition. In case of poor-quality images, or in case of partially saved slices of the scan set, as for model A2, the contour method is useful. The numerical results may not only depend on the mesh size but also on the domain configuration which can slightly vary according to the quality of the input data and the technique of the $3 \mathrm{D}$ reconstruction. The flow data must thus be used qualitatively, in order to improve the medical checkup, to help the physician to plane the treatment and to control the posttherapeutic vessel state. Variables of interest are estimated rather than properly quantified.

Literature data. Many studies have been performed on simple aneurism models to understand the main flow features in diseased region of vascular bed with either a side- or a branch-aneurism. Blood flow behaviour in an artery region with an aneurism has been shown to depend on (i) the vessel anatomy (local artery curvatures, branching configuration for branch aneurism, ...), (ii) on location, shape and size of the aneurism neck and sac and (iii) on the flow properties (local flow waveform, local values of the flow governing parameters, inflow angle, between-branch flow-rate ratio, ...). Cavity swirls, low dome velocity and flow separation in the transition zone of branching sites are the usual flow features in bulging region of the arterial network [14]. Both the artery wall and blood rheology have not been observed to be very important influencing factors [17]. Besides, flow features are different between steady and physiological flows, characterized by strong time gradients during the acceleration phase [24].

Because the geometry is one of the main factor affecting the flow in aneurisms, investigations must now be carried out in patient-specific domains derived from medical imaging in the context of 3D acquisition techniques $[4,20,24]$. Moreover, they must also use dynamical physiological input data. However, haemodynamic quantities are difficult to measure. Most often, pulsatile flowmetry is not associated with the angiography. In the present work, flow rates were measured in volunteers using cine phase-contrast MRI. In the future, radiologists will be asked to perform both angiography and flowmetry. But the accuracy of the flow variables remains questionable. The computer-aided selection of the vessel section and definition of the selected section contours of mid-size arteries, and, consequently, the flow-rate computation, are operator dependent.

\section{Conclusion}

Numerical results confirm what can be easily forecasted: a side aneurism have much lower risk of rupture than a branching-site one, either lateral or terminal, which is subjected by direct blood impact. Indeed, the flow is much more important in the cerebral aneurism, the neck of which is large and located in front of the vessel trunk axis, than in the side one. Consequently, the normalized pressure is higher in the terminal aneurism than in the side one. We can suppose that there is a risk of wall rupture where the aneurism pressure is high, assuming 
that the within-wall high stress concentration is correlated to high pressure. In the future, the Navier-Stokes equations must be coupled with vessel wall mechanics equations. However, one must note that there is a lack in suitable in vivo rheological properties of the vessel wall. Furthermore, blood-vessel wall interaction simulations are still in progress [10].

The intervention to prevent the aneurism rupture consists in implantation of coils to fill the pathological cavity. This catheter-based intervention is very difficult, because the physician must avoid rupture while filling the cavity and embolism due to a coil segment located too near the artery lumen. Once the operation ended, a residual cavity exists because of blood impaction and clotting retraction. The greater the residual cavity, the higher the development of a new aneurismal cavity. It is then important to fill correctly the high pressure zones of the aneurismal cavity. The computations can be helpful to plan coil implantation to minimize the risk. The aneurism sometimes compresses the artery and may require stenting associated with coiling, another endovascular therapy. Possible additional stenting can also benefit from local blood dynamic determination. Patient-specific numerical simulations will be required not only for a complete current state of the lesion and treatment planning but also for a lesion follow-up, especially after endovascular device settings.

Acknowledgements. Support for this work was provided by INRIA with ARC VITESV. Among involved people, we thanks S. Piperno for his help in computing the local axis at the vessel ends and R. Chaine, P. Frey, G. Malandain and E. Saltel for their help in image processing and meshing.

\section{REFERENCES}

[1] D.N. Arnold, F. Brezzi and M. Fortin, A stable finite element for the Stokes equations. Calcolo 21 (1984) 337-344.

[2] J.-D. Boissonnat and M. Yvinec, Algorithmic Geometry. Cambridge University Press, UK (1998).

[3] J.-D. Boissonat, R. Chaine, P. Frey, J.F. Gerbeau, G. Malandain, F. Nicoud, S. Salmon, E. Saltel and M. Thiriet, From medical images to computational blood flow models. INRIA Research Report (2003).

[4] V.D. Butty, K. Gudjonsson, P. Buchel, V.B. Makhijani, Y. Ventikos and D. Polikakos, Residence time and basins of attraction for a realistic right internal carotid artery with two aneurysms. Biorheology 39 (2002) 387-393.

[5] S. Chien, Shear dependence of effective cell volume as a determinant of blood viscosity. Science 168 (1970) 977-978.

[6] S. Chien, Biophysical behavior in suspensions, in The Red Blod Cell, D. Surgenor Ed., Academic Press, New York (1975).

[7] P.G. Ciarlet, The finite element method for elliptic problems. Stud. Math. Appl. 4 (1978).

[8] G.G. Ferguson, Physical factors in the initiation, growth and rupture of human intracranial saccular aneurysms. J. Neurosurg. 37 (1972) 666-677.

[9] P. Frey, A fully automatic adaptive isotropic surface remeshing procedure. INRIA Research Report 0252 (2001).

[10] J.-F. Gerbeau and M. Vidrascu, A Quasi-Newton Algorithm Based on a Reduced Model for Fluid-Structure Interaction Problems in Blood Flows. INRIA Research Report 4691 (2001).

[11] P.L. George, F. Hecht and E. Saltel, TetMesh (distributed by SIMULOG).

[12] R. Glowinski, Numerical methods for nonlinear variational problems. Springer Ser. Comput. Phys. (1984).

[13] F. Hecht and C. Parès, NSP1B3 : un logiciel pour résoudre les équations de Navier Stokes incompressible 3D. INRIA Research Report 1449 (1991).

[14] T.M. Liou and S.N. Liou, A review on in vitro studies of hemodynamic characteristics in terminal and lateral aneurysm models. Proc. Natl. Sci. Counc. ROC(B) 4 (1999) 133-148.

[15] W.E. Lorensen and H.E. Cline, Marching cubes: A high resolution 3D surface construction algorithm. Comput. Graphics 21 (1987) 163-169.

[16] J.-B. Mossa, Simulation d'une bifurcation artérielle. CERFACS Report (2001).

[17] K. Perktold, R. Peter and M. Resch, Pulsatile non-newtonian blood flow simulation through a bifurcation with an aneurysm. Biorheology 26 (1989) 1011-1030.

[18] O. Pironneau, On the transport-diffusion algorithm and its application to the Navier-Stokes equations. Numerische Mathematik 38 (1982) 309-332.

[19] H.J. Steiger, D.W. Liepsch, A. Poll and H.J. Reulen, Hemodynamic stress in terminal saccular aneurysms: a laser-Doppler study. Heart Vessels 4 (1988) 162-169.

[20] D.A. Steinman, J.S. Milner, C.J. Norley, S.P. Lownie and D.W. Holdsworth, Image-based computational simulation of flow dynamics in a giant intracranial aneurysm. AJNR Am. J. Neuroradiol. 24 (2003) 559-566

[21] G. Taubin, Curve and surface smoothing without shrinkage, in 5th Int. Conf. on Computer Vision Proc. (1995) $852-857$. 
[22] M. Thiriet, G. Martin-Borret and F. Hecht, Ecoulement rhéofluidifiant dans un coude et une bifurcation plane symétrique. Application à l'écoulement sanguin dans la grande circulation. J. Phys. III France 6 (1996) 529-542.

[23] M. Thiriet et al., Apports et limitations de la vélocimétrie par résonance magnétique nucléaire en biomécanique. Mesures dans un embranchement plan symétrique. J. Phys. III France 7 (1997) 771-787.

[24] M. Thiriet, P. Brugières, J. Bittoun and A. Gaston, Computational flow models in cerebral congenital aneurisms: I. Steady flow. Méca. Ind. 2 (2001) 107-118.

[25] M. Thiriet, S. Naili and C. Ribreau, Entry length and wall shear stress in uniformly collapsed-pipe flow. Comput. Model. Engrg. Sci. 4 (2003) No. 3 and 4.

To access this journal online:

www.edpsciences.org 